\title{
Internetwork magnetic field as revealed by two-dimensional inversions
}

\author{
S. Danilovic ${ }^{1}$, M. van Noort ${ }^{1}$, and M. Rempel ${ }^{2}$ \\ 1 Max-Planck-Institut für Sonnensystemforschung, Justus-von-Liebig-Weg 3, 37077 Göttingen, Germany \\ e-mail: danilovic@mps.mpg.de \\ ${ }^{2}$ High Altitude Observatory, NCAR, PO Box 3000, Boulder, Colorado 80307, USA \\ Received 26 November 2015 / Accepted 23 June 2016
}

\begin{abstract}
Context. Properties of magnetic field in the internetwork regions are still fairly unknown because of rather weak spectropolarimetric signals.

Aims. We address the matter by using the two-dimensional (2D) inversion code, which is able to retrieve the information on smallest spatial scales up to the diffraction limit, while being less susceptible to noise than most of the previous methods used.

Methods. Performance of the code and the impact of various effects on the retrieved field distribution is tested first on the realistic magneto-hydrodynamic (MHD) simulations. The best inversion scenario is then applied to the real data obtained by Spectropolarimeter (SP) on board Hinode.

Results. Tests on simulations show that: (1) the best choice of node position ensures a decent retrieval of all parameters; (2) the code performs well for different configurations of magnetic field; (3) slightly different noise levels or slightly different defocus included in the spatial point spread function (PSF) produces no significant effect on the results; and (4) temporal integration shifts the field distribution to a stronger, more horizontally inclined field.

Conclusions. Although the contribution of the weak field is slightly overestimated owing to noise, 2D inversions are able to recover well the overall distribution of the magnetic field strength. Application of the 2D inversion code on the Hinode SP internetwork observations reveals a monotonic field strength distribution. The mean field strength at optical depth unity is $\sim 130 \mathrm{G}$. At higher layers, field strength drops as the field becomes more horizontal. Regarding the distribution of the field inclination, tests show that we cannot directly retrieve it with the observations and tools at hand, however, the obtained distributions are consistent with those expected from simulations with a quasi-isotropic field inclination after accounting for observational effects.
\end{abstract}

Key words. Sun: magnetic fields - Sun: photosphere - techniques: polarimetric - techniques: spectroscopic

\section{Introduction}

Determining the magnetic properties of the internetwork has always been an important task (Sánchez Almeida \& Martínez González 2011) because it carries a substantial portion of the solar magnetic flux and has a large impact on the energy budget in the solar atmosphere. For decades it has been clear that the internetwork is filled with magnetic field elements of opposite polarities organized on small scales (Livingston \& Harvey 1975; Livi et al. 1985; Martin 1988). Yet, whether their field strength is predominantly $\mathrm{hG}$ or $\mathrm{kG}$, i.e., how much flux they carry, has been the subject of debate (Keller et al. 1994; Lin 1995; Sánchez Almeida \& Lites 2000; Socas-Navarro \& Lites 2004; Domínguez Cerdeña et al. 2006; Martínez González et al. 2006).

As the polarimetric sensitivity and spatial resolution of the observations increased, it became possible to characterize the full magnetic field vector. The debate then expanded further on the inclination of the magnetic field. Currently, three hypotheses remain: the internetwork magnetic field is predominantly horizontal (Orozco Suárez et al. 2007a,b; Lites et al. 2008), predominantly vertical (Beck \& Rezaei 2009; Stenflo 2010; Ishikawa \& Tsuneta 2011), or quasi-isotropic (Asensio Ramos 2009; Asensio Ramos \& Martínez González 2014).

The basic problem lies in retrieving information on magnetic field based on the rather weak internetwork spectropolarimetric signals. The influence of noise on the result is overwhelming and leads to a systematic overestimation of the inclination of the magnetic field vector (Borrero \& Kobel 2011). One way of getting around that problem is to limit the analysis only to pixels where the signal-to-noise ratio is high enough. These selection criteria tend to exclude a significant portion of the internetwork surface, however, and to bias the retrieved distributions of magnetic field strength and inclination in different ways (Borrero \& Kobel 2012).

In this paper, we address the issue via the $2 \mathrm{D}$ inversion technique (van Noort 2012) that accounts for spatial coupling between the neighboring pixels and simultaneously and selfconsistently fits the observed spectra, which makes it less susceptible to noise than most of the previous methods used. The code is first tested on synthesized observations produced from realistic magnetohydrodynamic simulations, and then the best inversion strategy is applied to real observations. We limit our study to the general properties of the distributions of the magnetic field strength and inclination.

\section{Two-dimensional inversions}

To invert a Hinode Spectropolarimeter (SP) map of the quiet Sun, we used the SPINOR inversion code (Frutiger 2000; Frutiger et al. 2000). This inversion code fits a strongly simplified atmosphere using one or more spectral lines with the 
Table 1. Main characteristics of the MHD snapshots used to test the inversion code.

\begin{tabular}{lllll}
\hline \hline Name & Dimension & Resolution & $\begin{array}{l}\text { Longitudinal } \\
\text { flux density }\end{array}$ & $\begin{array}{l}\text { Transverse } \\
\text { flux density } \\
{[\mathrm{G}]}\end{array}$ \\
& {$[\mathrm{Mm}]$} & {$[\mathrm{km}]$} & {$[\mathrm{G}]$} & 54.2 \\
\hline Sim 1 & $4.86 \times 4.86 \times 1.4$ & $5 \times 5 \times 7$ & 4.2 & 52.8 \\
Sim 2 & $24.6 \times 24.6 \times 7.68$ & $16 \times 16 \times 16$ & 10.5 & 53.1 \\
Sim 3 & $6 \times 6 \times 1.68$ & $10 \times 10 \times 14$ & 6.8 & 53 \\
\hline
\end{tabular}

Notes. Mean unsigned longitudinal and transverse flux densities are calculated after all the instrumental effects are taken into account.

assumption of local thermodynamic equilibrium (LTE). The atmosphere is described by the values of a selectable number of atmospheric quantities, such as temperature, line-of-sight (LOS) velocity, magnetic field strength, and direction, and microturbulent broadening, which is specified at a selectable number of optical heights, the so-called node positions. The code is used in its spatially coupled mode (van Noort 2012), in which the accurately known spatial point spread function (PSF) of the Hinode Solar Optical Telescope (SOT) is taken into account when the observed data are fitted.

To be able to reach the diffraction limit of Hinode SOT, we oversampled all Stokes maps by a factor two, to $0.08^{\prime \prime}$, following van Noort et al. (2013). Taken that the current version of the code can only use shared memory, the size of the input map is limited to $200 \times 200$ pixels. The final maps were then collected as mosaics of individually inverted smaller maps.

Since the model atmosphere used in previous studies (van Noort et al. 2013; Riethmüller et al. 2013; Tiwari et al. 2013; Lagg et al. 2014; Bühler et al. 2015) proved to provide a good fit even to very complex profiles, we also used this model here. The model describes a height dependent atmosphere at three nodes in optical depth with the following free parameters: temperature, magnetic field strength, magnetic field inclination with respect to the LOS, azimuth of the magnetic field vector, LOS velocity, and a microturbulent velocity. The inversion strategy is also the same as in the previous studies. The code was run for ten iteration steps, followed by a spatial smoothing of the fitted atmosphere, the result of which was then used as the input for the next ten iteration steps. This cycle was repeated eight times before the result was considered fully converged. To make sure that the result was really converged, the cycle was repeated up to 20 times for certain maps, but these additional cycles brought no significant improvements to the mean $\chi^{2}$ value.

\section{Simulations}

To test our inversions, we used three snapshots from three different runs produced with the MURaM code (Vögler et al. 2005). Since we are interested in finding out what the inversion process does with weak quiet Sun signals, we chose snapshots that produce spectropolarimetric signals of similar strength. Given that the origin of the quiet Sun field is still a subject of debate, we took two snapshots from the local dynamo runs and one where pure flux emergence is taking place. The field configurations in all three simulations are predominantly horizontal. Although some studies suggest the real quiet Sun may have a predominantly vertical field (Stenflo 2010; Ishikawa \& Tsuneta 2011), we were not able to produce a snapshot with such field configuration that matches the observed level of linear polarization as well as circular polarization signals. Hence, we did not include this type of simulation in the test. The main properties of the chosen snapshots are given in Table 1. The maps of the intensity and magnetic field parameters in all three snapshots are given in Fig. 1.

The first snapshot, Sim 1, is a snapshot from the saturated phase of Run $\mathrm{G}\left(R_{m} \sim 5200\right)$ from Danilovic et al. (2010). A detailed description of these dynamo simulations is given in Vögler \& Schüssler (2007) and Pietarila Graham et al. (2010). To get the observed level of spectropolarimetric signals, we multiplied the field strength with a factor of 2 , following Danilovic et al. (2010). Sim 2 is a snapshot from a more realistic dynamo simulations that tries to take into account the contribution coming from the large-scale dynamo by introducing an open bottom boundary. This snapshot is a non-gray version of the run O16bM from Rempel (2014). The photosphere is located about $1.5 \mathrm{Mm}$ beneath the top boundary. The open bottom boundary allows for the presence of a small-scale horizontal magnetic field in upflow regions in an attempt to mimic a deep magnetized convection zone (Rempel 2014). The last snapshot, $\operatorname{Sim} 3$, is taken from a run that simulates pure flux emergence with no local dynamo action present. The run is similar to the run used in Danilovic et al. (2015) with the difference that a horizontal flux sheet is introduced into a purely hydrodynamic run. The field strength in the flux sheet is set to vary across the cross section as a Gaussian with a width of $110 \mathrm{~km}$ and a maximum value of $300 \mathrm{G}$. The initial position of the sheet is some $300 \mathrm{~km}$ below the height where the mean optical depth is unity. We took a snapshot some $15 \mathrm{~min}$ into the run.

All three snapshots were treated in the same way to produce synthetic observations. Hinode spectropolarimetric observations were simulated by convolving with an appropriate spectral and spatial PSF (Danilovic et al. 2008). Also Gaussian noise at the level of $8 \times 10^{-4} I_{\mathrm{c}}$ was added to simulate deep magnetogram observations. Table 1 shows the longitudinal and transverse flux densities for every snapshot calculated with the application of the solarsoft routines (Lites \& Ichimoto 2013) on the artificial data after the convolutions were applied. The values are very close to those found in observations (Danilovic et al. 2010) ${ }^{1}$. The final preparatory step was the interpolation of such obtained maps to a finer sampling, as for real observations.

\section{Tests on simulated Hinode data}

van Noort (2012) showed that the 2D inversions are able to retrieve all physical parameters when the input data are spectra computed from simplified atmospheres. Here, we determined how far the final result is off if we feed the inversions with spectral profiles calculated from the complex original stratification of magneto-hydrodynamic (MHD) simulations. We are not interested in the accuracy of the retrieved values of individual pixels. We are, however, interested in the overall distribution of the key

The values for Sim 1 are obtained after the field strength was scaled. 

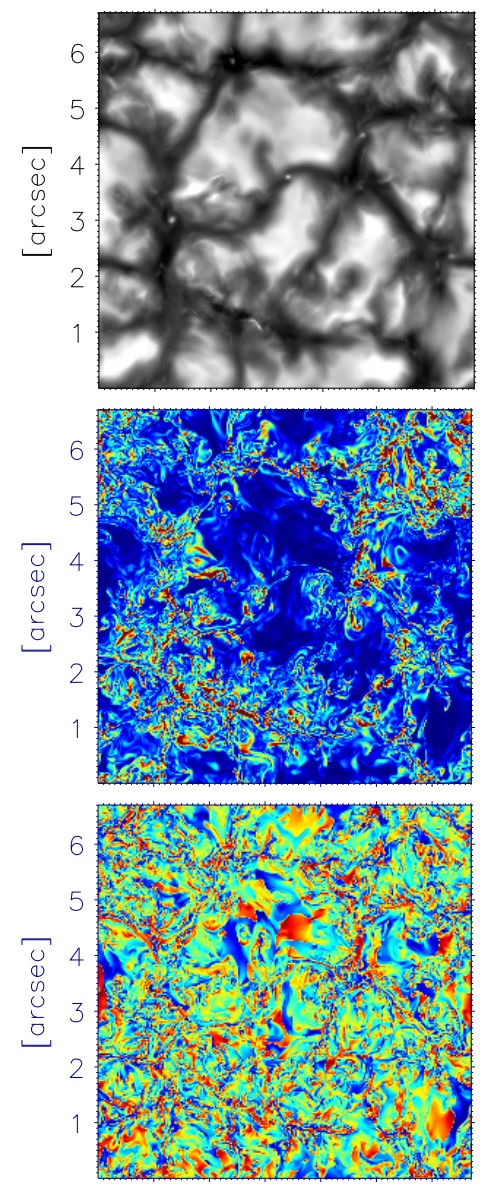

$$
\left[\begin{array}{cc}
3 & 4 \\
\text { arcsec }
\end{array}\right]
$$
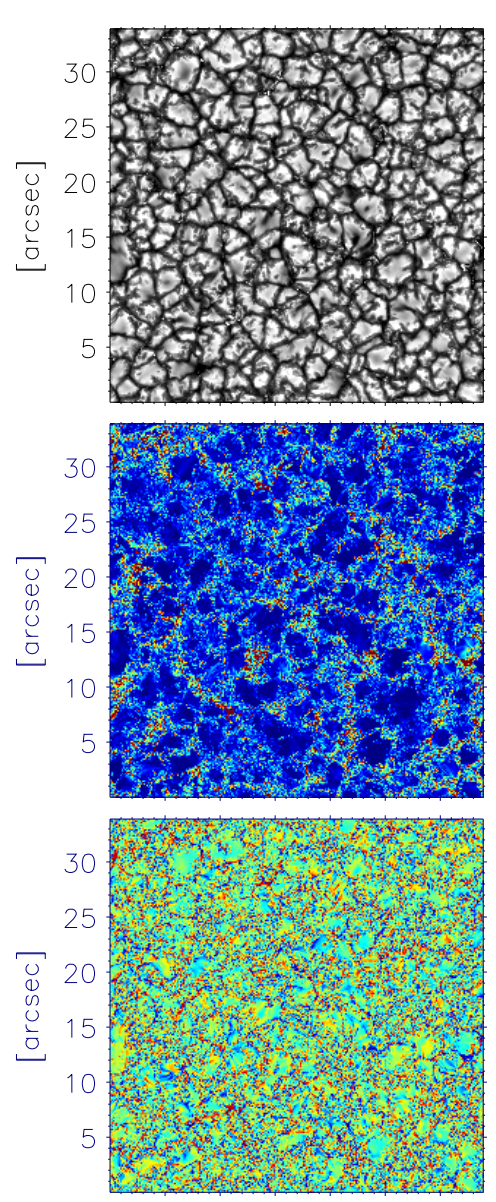

[arcsec]
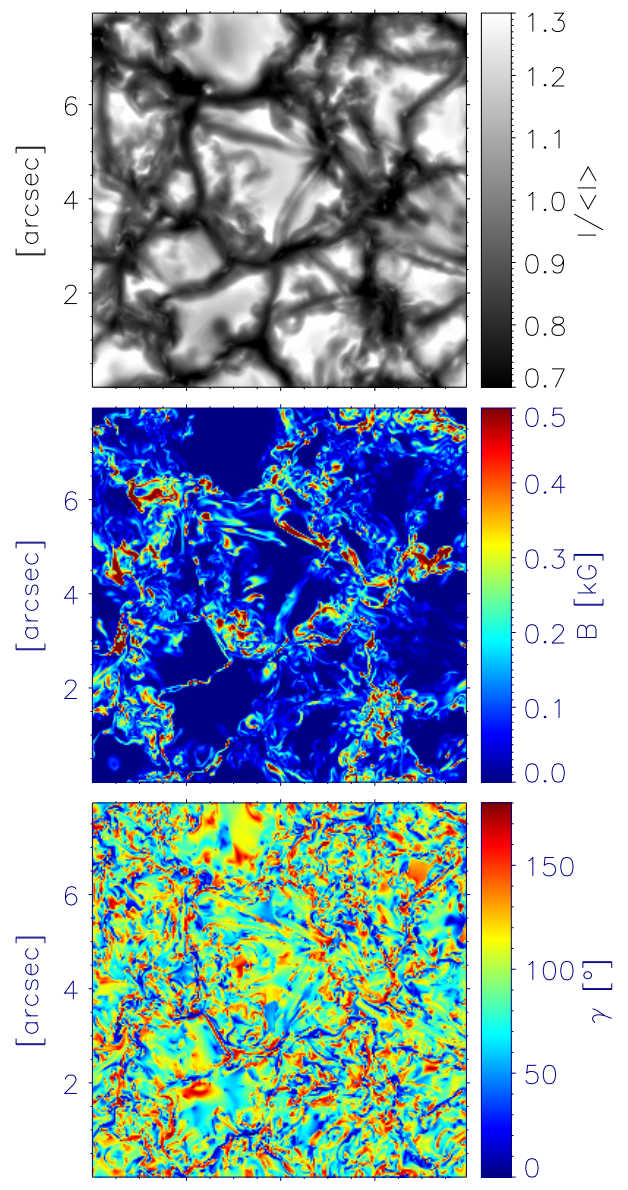

$$
\text { [arcsec] }
$$

Fig. 1. Overview of the MHD snapshots used for testing 2D inversions: intensity (top row), magnetic field strength (middle row), and inclination (bottom row) in Sim 1 (left), Sim 2 (middle), and Sim 3 (right) at $\log \tau=0$.

parameters, i.e., velocity, magnetic field strength, and inclination. To make a quantitative measure of the ability of the inversion code to retrieve these physical parameters, one first needs to smear the original simulation somehow to the resolution of the simulated observations. Since the 2D inversions should retrieve only spatial frequencies lower than the diffraction limit of the Hinode SOT, we degraded the original maps for every parameter at a constant optical depth with a low-pass filter with a cutoff at $3.85 \mathrm{arcsec}^{-1}$ and then rebinned to the pixel size of the oversampled observations $\left(0.08^{\prime \prime}\right)$. The inclination maps were treated differently because the spatial averaging in this case was weighted with the field strength. Since Stokes $V$ signal scales linearly with longitudinal component $B_{\text {long }}$, and $Q$ and $U$ scale quadratically with transverse component $B_{\text {tra }}$ of the magnetic field, smearing in Stokes space makes the derived fields more horizontal because of the different scaling. We thus applied the filter to $B_{\text {long }}$ and $B_{\text {tra }}^{2}$ separately, rebinned them, and then computed the inclination for every resized pixel. Treating original maps in this way enabled us to make a pixel-to-pixel comparison of the retrieved and original simulated parameter as a function of optical depth. Figure 2 shows the example for a snapshot from Sim 2. The spatial filtering seems to change the appearance of the inclination map the most.

The parameter that is retrieved most robustly in Fig. 2 is the velocity. The inverted velocity map retains all the details visible in the original maps, i.e., the small downflow in the center of the granule at $\left[23^{\prime \prime}, 17^{\prime \prime}\right]$ or the strong upflow at the edge of the granule at $\left[5^{\prime \prime}, 21^{\prime \prime}\right]$. Apart from being noisy, the inverted map of magnetic field strength looks very much like the original. The strong field features together with patches of no field are recovered well. In places, though, the field strength is a bit higher, which is probably because of the noise included in the simulated observations. The inclination, on the other hand, seems to reveal more horizontal field than is really present. The areas where the inversion code misses the small-scale mixed polarity correspond to regions where the field strength is very weak. The inversion code, however, retrieves the inclination of the stronger magnetic features well.

Achieving this result required some testing, which we present below. We investigated how the results depend on the setup of the node positions in the inversions, input configuration of the magnetic field, choice of a wrong PSF, noise level, and evolution of the underlying solar scene.

\subsection{Node position setup}

Our tests show that the distribution of inverted parameters varies the most with the changes in inversion node positions. We tried various combinations of which we only show the extreme cases to illustrate the amount of scatter. Figure 3 shows the difference between the input and inverted values as a function of optical depth and demonstrates how much systematic error is introduced 


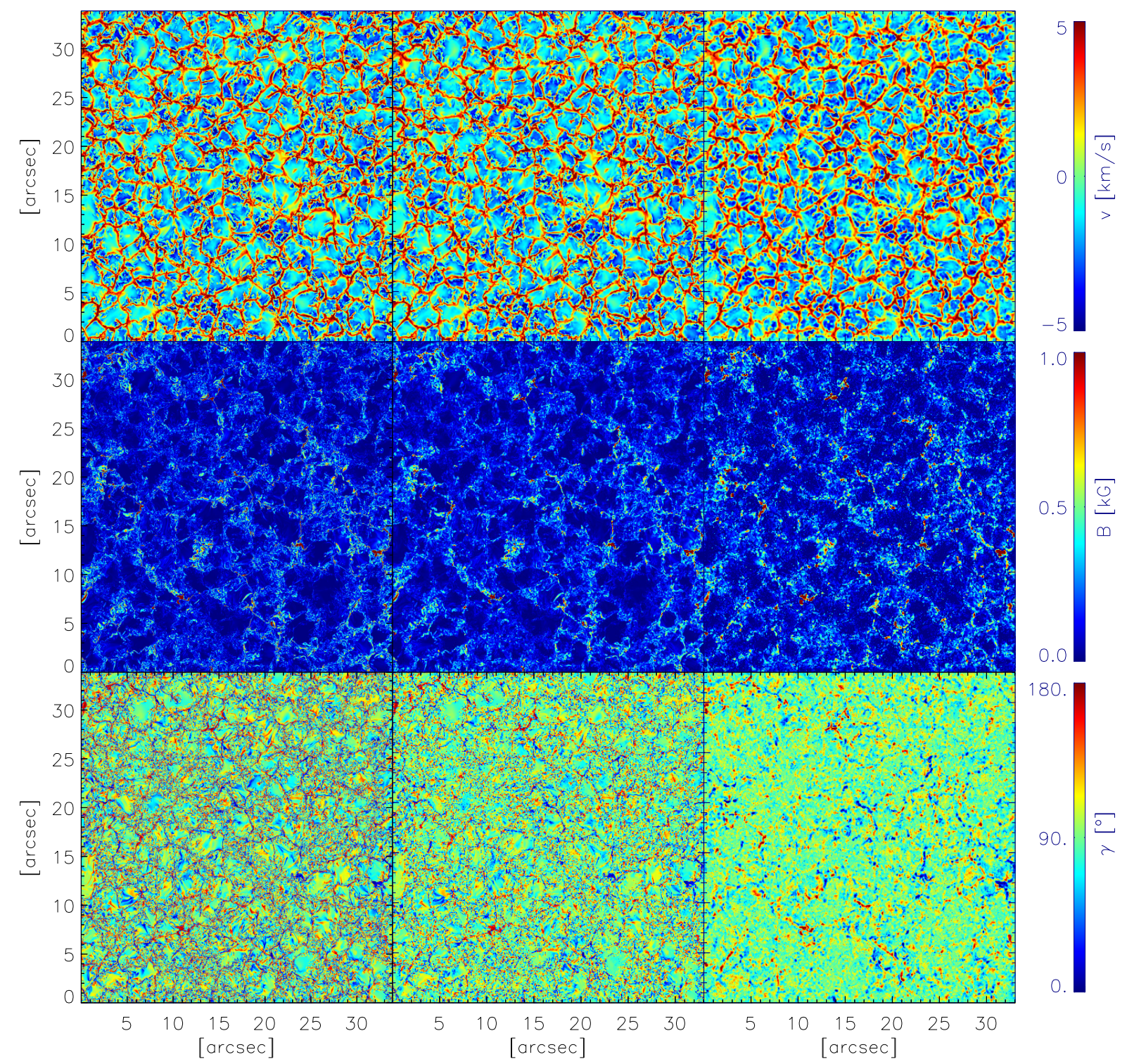

Fig. 2. Results of $2 \mathrm{D}$ inversions applied to simulations; comparison of maps of velocity (top row), magnetic field strength (middle row) and inclination (bottom row) at $\log \tau=0$ in Sim 2. Three columns, from left to right, show the original unsmeared maps from the snapshot, the same maps after the highest spatial frequencies are filtered out, and the results from the inversions.

when only the top node is shifted from $\log \tau=-1.5$ to $\log \tau=$ -2.5. The lower two nodes are kept fixed at $\log \tau=0$ and -0.8 .

As shown by van Noort (2012), the velocity is a pretty robust quantity, whose determination is not very sensitive to noise since it can be basically inferred directly from the intensity. It is well recovered in all layers of the atmosphere with a mean error that is always smaller than $20 \mathrm{~m} / \mathrm{s}$. The best match to the original value, in the lower as well as upper atmosphere, is obtained when the top node is placed at $\log \tau=-2.0$. Shifting the top node upward or downward gives underestimated or overestimated velocities at the surface, respectively. Velocities in the upper atmosphere are underestimated in both cases.

Although the determination of the magnetic field strength is more prone to noise induced errors, Fig. 3 shows that a pixel-topixel comparison yields an accumulation of points around 0 . The mean error in the top layers ranges from close to $100 \mathrm{G}$ when the top node is set to $\log \tau=-2.5$ to less than $20 \mathrm{G}$ when the top node is set to $\log \tau=-2.0$. All three curves come the closest to zero around the middle node. This is not a surprise given that the inversions are most sensitive and accurate at this height when the Fe I $630 \mathrm{~nm}$ lines are fitted (Cabrera Solana et al. 2005).

A pixel-to-pixel comparison of the original and inverted values shows the largest scatter for the magnetic field inclination. The standard deviation curves exceed $30^{\circ}$ in all three cases, peaking when the top node is at $\log \tau=-1.5$. Shifting the middle node from $\log \tau=-0.8$ to -1 gives a larger mean error at higher layers of the atmosphere. It is even doubled for some quantities.

For further tests, we used the optimum combination of node positions at $\log \tau=0,-0.8$ and -2.0 .

\subsection{Difference in the magnetic field configuration}

Since all of the snapshots give a similar amount of spectropolarimetric signal, as shown in Table 1, all three have a similar field strength distribution. A slight difference exists in the upper atmosphere where Sim 1 has significantly lower field strength than the other two snapshots. This is because this early dynamo run contains predominantly small-scale magnetic field loops that 

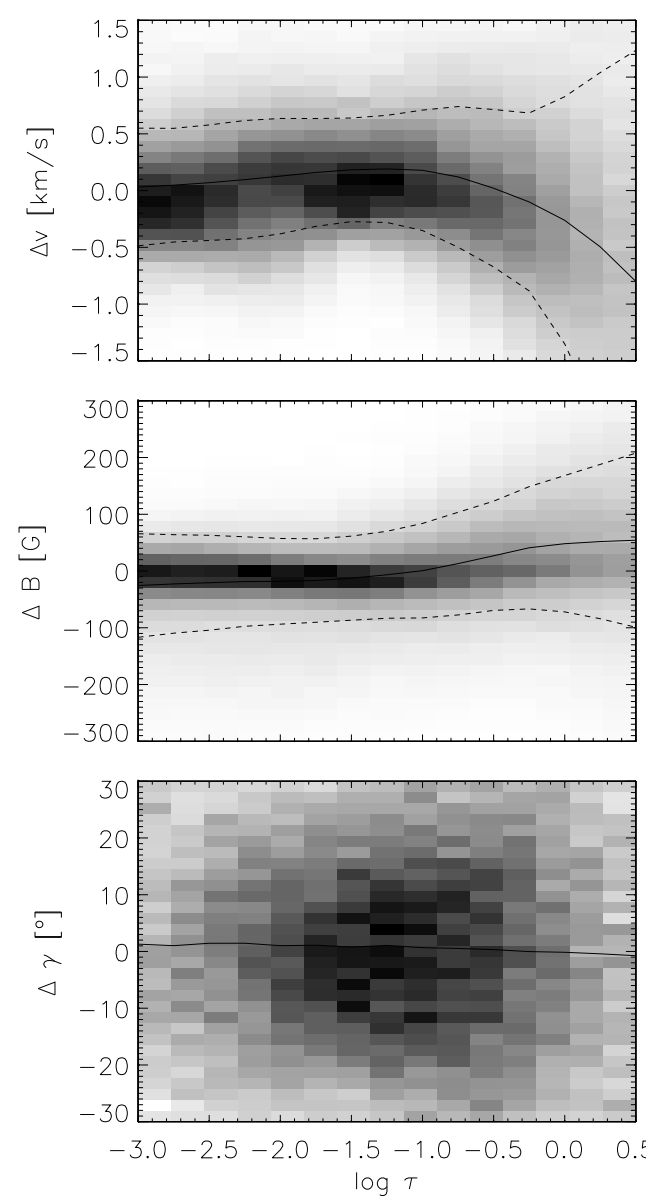
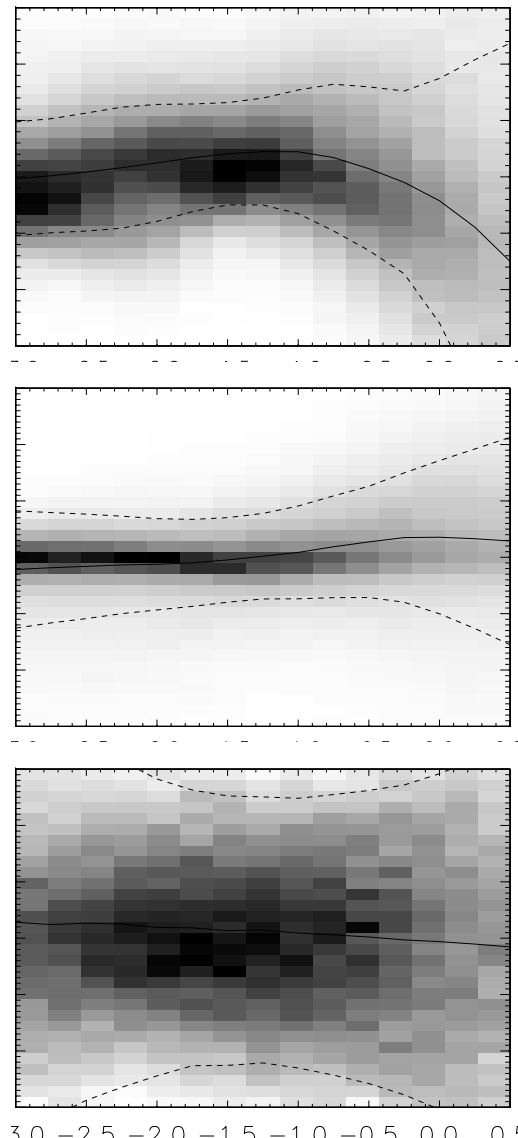

$\log$
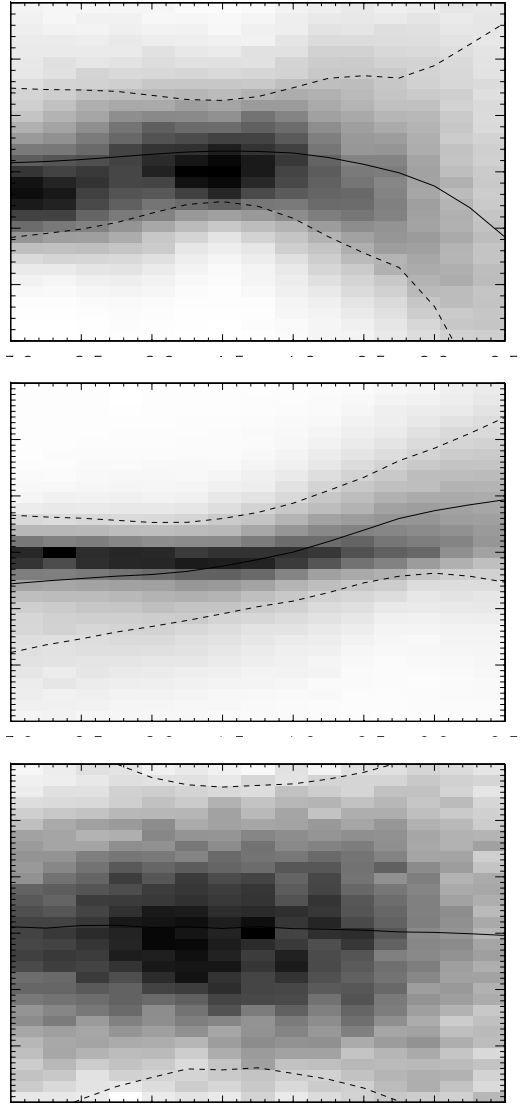

$0-2.5-2.0-1.5$

Fig. 3. Results of 2D inversions applied to simulations; test of node position in Sim 1 when the top node is placed at log $\tau=-1.5$ (left column), $\log \tau=-2.0$ (middle column), and $\log \tau=-2.5$ (right column). Two-dimensional histograms of the difference of the original smeared and inverted quantities: magnetic field (top), LOS velocity (middle), and inclination (bottom) as a function of optical depth. The mean difference is indicated by solid lines and the standard deviation of the scatter is indicated by the dashed.

close low down (Schüssler \& Vögler 2008), so the height profile of the mean field strength in this run drops off more rapidly than in the other two cases. Another difference is that Sim 2 is the only snapshot that shows a large-scale magnetic field distribution and, hence, shows more strong field features at the solar surface than the other two examples.

The main difference is, however, the field entanglement, which is the main reason why these runs were chosen. The complexity of the field configuration increases with the resolution of the simulations. So, while Sim 3 has well-resolved magnetic features, Sim 1 and 2 show a small-scale salt and pepper pattern of opposite polarities that are characteristic of all local dynamo runs. Therefore, this test determines how much detail our 2D simulations are able to retrieve.

Figure 4 again shows the difference between the initial and retrieved parameters as a function of optical depth for all three cases. The 2D histograms look smoother for Sim 2 because of the larger domain, which gives better statistics. We give absolute rather than relative values, since the quantities do not differ significantly in magnitude for all three simulations. The figure demonstrates that the inversions behave similarly in all cases. The systematic errors are very similar for all three parameters.

What do the retrieved distributions look like? Figure 5 shows histograms of the original and retrieved magnetic field strength and inclination for all three snapshots. For clarity, we choose to give here only distributions at optical depth unity. This height is also the most interesting, since the parameters change on the smallest scales.

In the case of magnetic field strength, the retrieved distributions are very close to the original distributions generated after the spatial filtering is applied and the distribution tail at the highest field strengths is cut off. At the lowest values, the peaks at 5-20 G, which are visible in the original distributions, are lost after the $2 \mathrm{D}$ inversions because the inversions tend to set the field strength to 0 if no significant signal is found. Also, owing to noise, the inversions tend to overestimate the field strength for a large number of pixels, which is most obvious in the case of Sim 2, where the sample is the largest (largest field of view). This is further demonstrated in Fig. 7.

Vertical lines in Fig. 5 show the mean values for the corresponding field strengths. Retrieved numbers are fairly close to the original after the spatial filtering is applied. Because of the effects of noise, which tend to overestimate the weak field, the mean inverted values are slightly higher for all simulations. The same trend is present for all node positions. The deference is always the largest for Sim 3 where rapid emergence is simulated over whole simulation domain and the line-of-sight atmospheres are very complicated with large gradients in the field vector and velocity.

Inspection of the inclinations shows that the spatial smearing also removes a significant number of pixels with inclined or completely vertical field. After the inversions, the distributions 

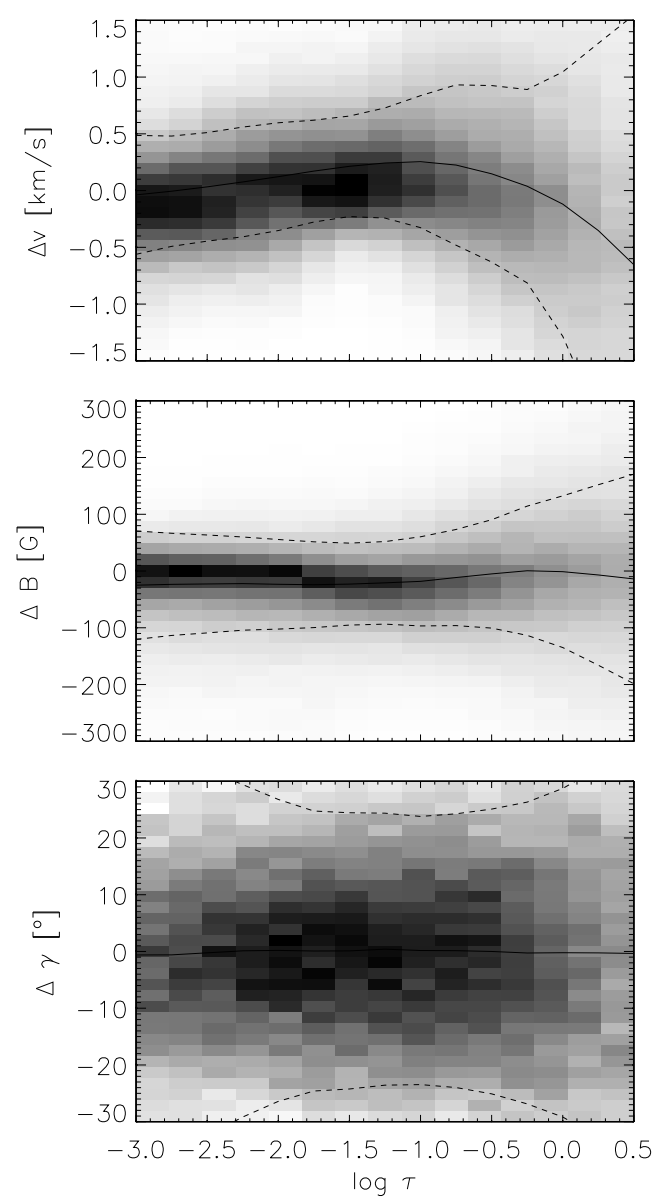
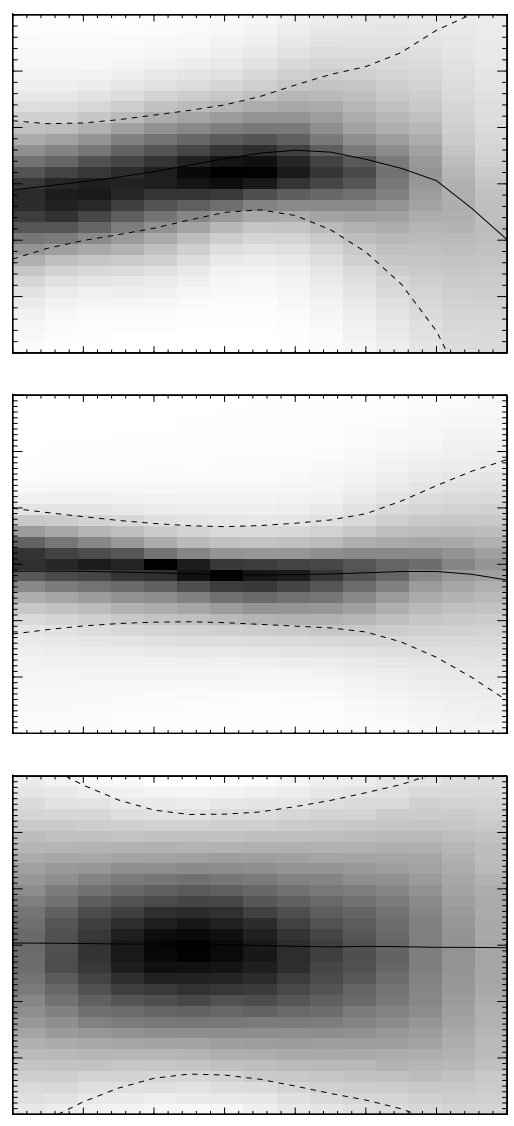

$\log \tau$
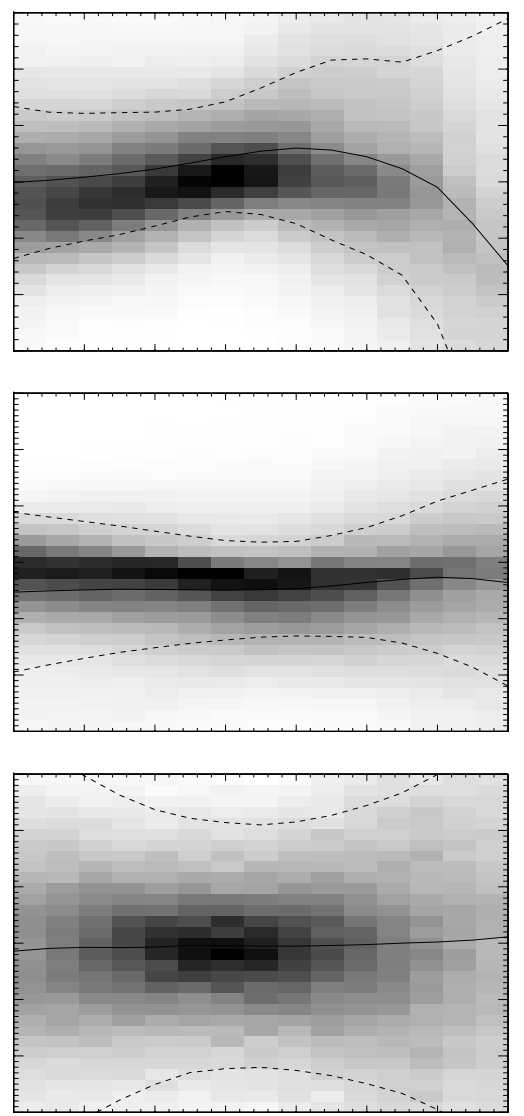

$\log \tau$

Fig. 4. Results of 2D inversions applied to simulations; test on different simulations: difference of original and inverted velocity (top row), magnetic field strength (middle row), and inclination (bottom row) as a function of optical depth for Sim 1, 2, and 3 from left to right.
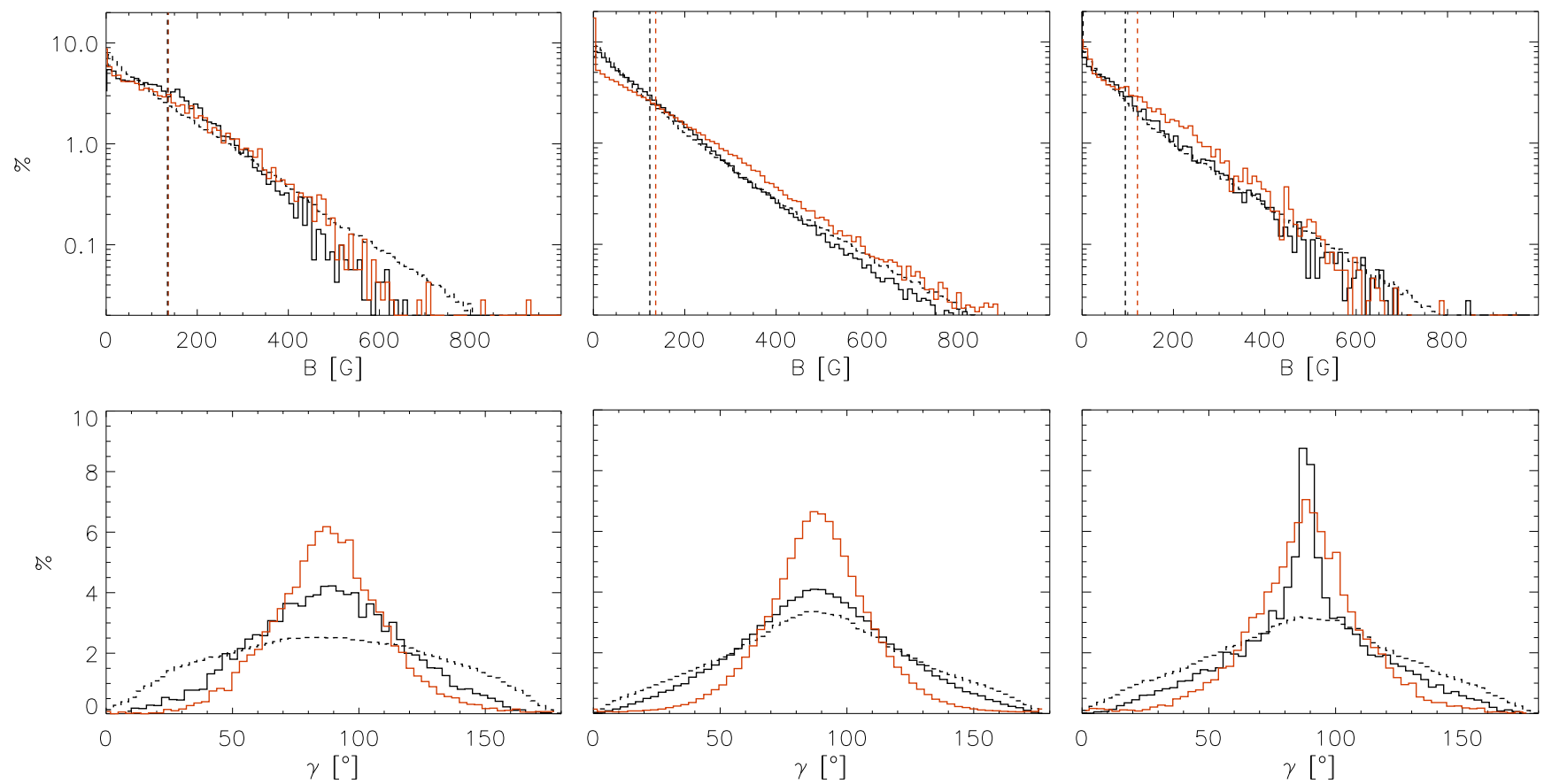

Fig. 5. Results of 2D inversions applied to simulations; test on different simulations: histograms of magnetic field strength (top row) and inclination (bottom row) at $\log \tau=0$ for Sim 1, 2, and 3 from left to right. Black dashed and solid lines indicate the original distributions before and after the spatial smearing, respectively. Red line shows distributions retrieved with $2 \mathrm{D}$ inversions. Bin sizes are $10 \mathrm{G}$ and $3^{\circ}$. Vertical lines indicate the corresponding mean field strengths at $\log \tau=0$ : from the original when the highest spatial frequencies are filtered out (black dashed line) and retrieved with inversions (red dashed line). 

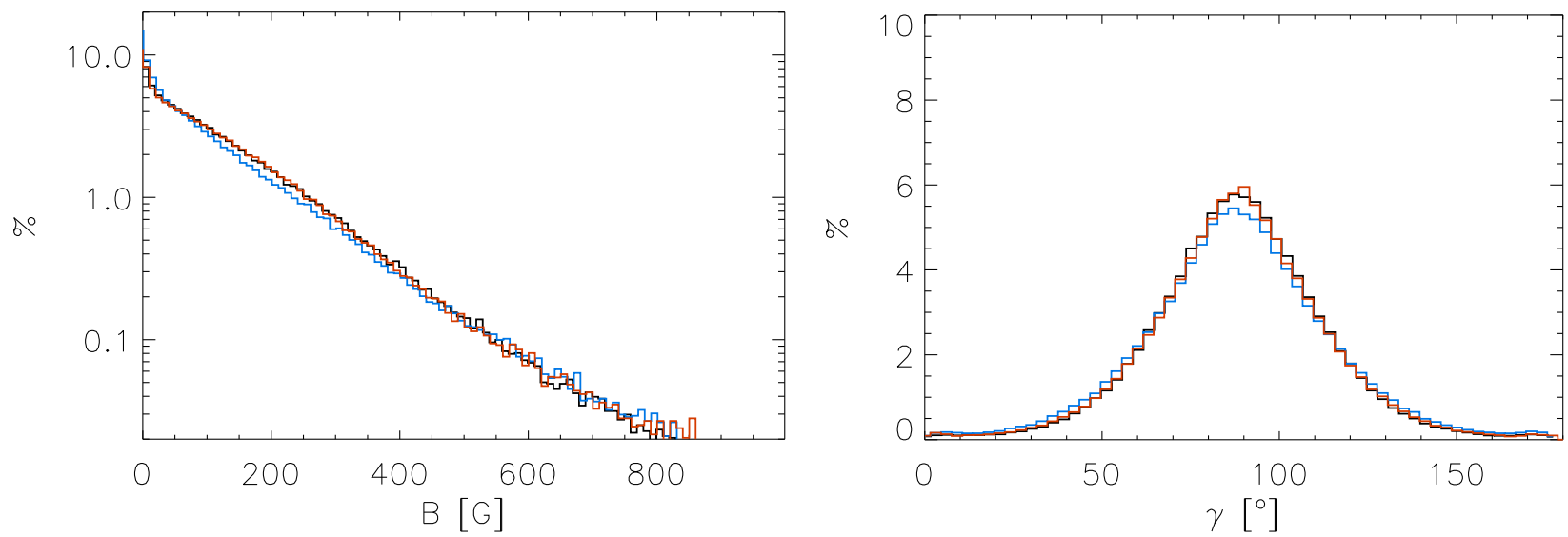

Fig. 6. Results of 2D inversions applied to simulations; effect of used PSF: histograms of magnetic field strength (left) and inclination (right) at $\log \tau=0$ for Sim 2 when different PSFs are used in 2D inversions. Black line represents the distributions when the same PSF is used to smear and retrieve the parameters. Blue and red lines show what happens when PSF used by 2D inversions assumes too little or too much of the defocus, respectively. Bin sizes are $10 \mathrm{G}$ and $3^{\circ}$.
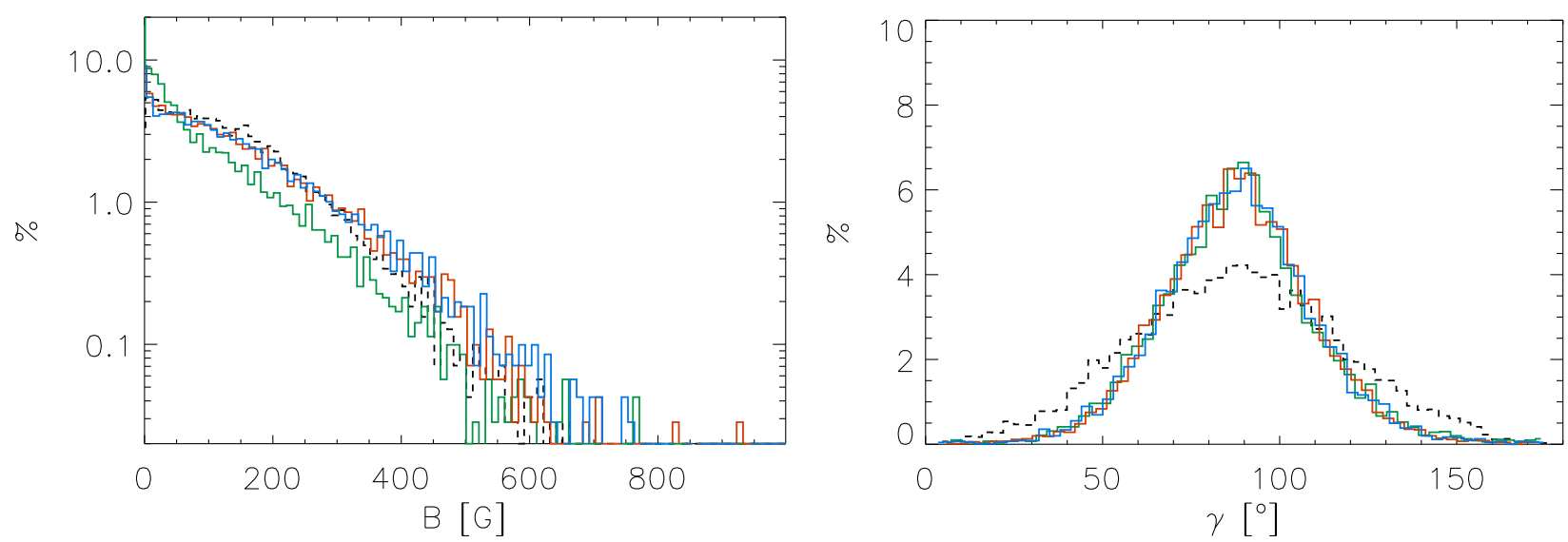

Fig. 7. Results of 2D inversions applied to simulations; effects of different noise level: histograms of magnetic field strength (left) and inclination (right) at $\log \tau=0$ for Sim 1 retrieved with 2D inversions. Green, red, and blue lines correspond to noise levels of $0,8 \times 10^{-4} I_{\mathrm{c}}$, and $1.2 \times 10^{-3} I_{\mathrm{c}}$, respectively. Black dashed lines denote the original distributions after the highest spatial frequencies were removed. Bin sizes are $10 \mathrm{G}$ and $3^{\circ}$.

are additionally modified to show more horizontal field, especially in the regions where the field strength is very weak, as shown in Fig. 2. The change in the distribution is somewhat less severe for Sim 3, which does not show the salt and paper pattern visible in the local dynamo simulations.

\subsection{Choice of PSF}

Owing to the Hinode SOT design, the Hinode SP observations were often taken with a significant departure from the best focus for the instrument (Lites 2011; Bühler et al. 2013). The combination of this somewhat random shift with the long-term drift of the best focus position makes the determination of the precise focus departure troublesome. This is why we tested how much the error in the defocus of the PSF used in our inversion effects the final result. We used three PSFs with different amount of defocus. The first PSF, in which the defocus of seven focus steps was included (Danilovic et al. 2008), was also used for degrading the synthetic images. In the other two PSFs, the defocus was underestimated or overestimated by four steps.

The test shows that the disagreement between the cases is hardly visible, so we omit the figure with the parameter differences as a function of optical depth. The systematic error in the velocity is slightly higher for the PSF where the defocus is underestimated, but the mean error, even then, is not larger than $30 \mathrm{~m} / \mathrm{s}$. When the PSF with too little defocus is used, the mean field strength at the solar surface is underestimated by less than $10 \mathrm{G}$. Too much defocus, on the other hand, overestimates the field by only a few G more than the "right" PSF. While the systematic error does not go over $10 \mathrm{G}$ when the proper PSF is used, too much defocus increases the mean error to $20 \mathrm{G}$ in the upper atmosphere.

Figure 6 shows how small the differences in the final inverted distributions really are. The difference is the largest in the weak field part of the field strength distribution. The PSF with an amount of defocus that is underestimated tends to also underestimate the contribution of the pixels with a weak field. On the other hand, assuming that defocus was larger than was actually the case slightly increases the number of pixels with weak, horizontal fields. All in all, the impact is statistically negligible.

\subsection{Noise level}

High-resolution Hinode SP scans are also taken in deep magnetogram mode that shifts the level of noise from $1.2 \times 10^{-3} I_{\mathrm{c}}$ (for normal mode) to $8 \times 10^{-4} I_{\mathrm{c}}$. To check if this improves the 

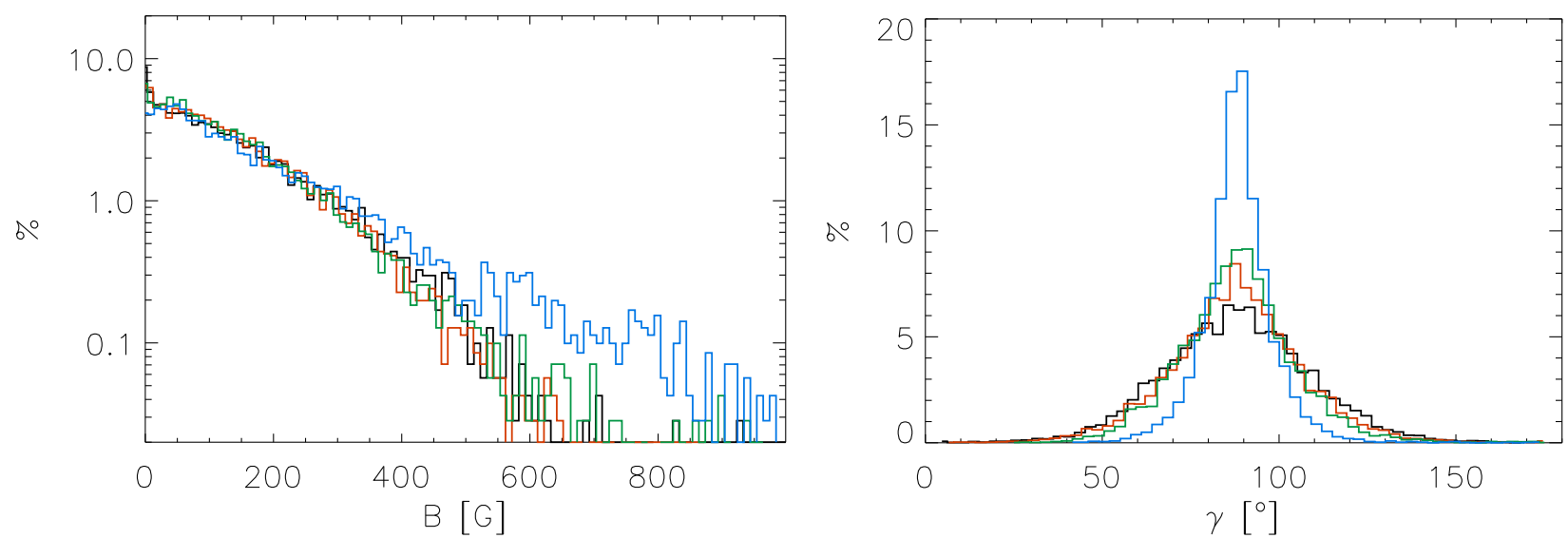

Fig. 8. Results of 2D inversions applied to simulations; effect of temporal integration: histograms of magnetic field strength (left) and inclination (right) at $\log \tau=0$ for Sim 1 retrieved with 2D inversions. Black, red, green, and blue lines correspond to cases where we intergrated over $0 \mathrm{~s}$, $20 \mathrm{~s}, 2 \mathrm{~min}$, and $9 \mathrm{~min}$, respectively. Bin sizes are $10 \mathrm{G}$ and $3^{\circ}$.

results, we simulated both cases. Figure 7 shows the resulting distributions of magnetic field strength and inclination retrieved with 2D inversions. There seems to be no significant difference. The result stays almost the same even if no noise is included. The figure suggests that impact of noise is in redistributing the pixels with very weak field to hG field bins and generally making the field stronger. The distribution of inclination does not benefit from lack of noise either. The problem is intrinsically in a simple model we assume, which tries to match very asymmetric Stokes profiles produced by complex MHD atmospheres.

\subsection{Solar photospheric evolution}

For all the previous tests, we used one snapshot to simulate the observations taken with a finite exposure time. Here, we tested how much the results change if we take into account the actual evolution of magnetic features during this time. Because the simulations where emergence takes place would lead to an amount of horizontal field that increases with time, we take Sim 1, where the flux stays almost constant. Simulating a proper Hinode SP scan requires a large set of snapshots, produced with very high cadence. Given that these simulation cover only $6^{\prime \prime} \times 6^{\prime \prime}$ that Hinode SP scans in only $3 \mathrm{~min}$ in the normal mode, we found that integrating for every pixel over the whole box would be a close enough approximation that would produce a similar effect as averaging for every slit position separately. We chose to integrate over $20 \mathrm{~s}, 2 \mathrm{~min}$ and $9 \mathrm{~min}$. Every snapshot within this period was then treated the same. Spatial smearing and adding the noise of $8 \times 10^{-4} I_{\mathrm{c}}$ was applied first to each of them and then temporal integration of the resulting signal was performed separately for every rebinned pixel and every wavelength point. In this way, the intensity contrast is reduced by $16 \%$ and apparent longitudinal flux density decreased by $37 \%$.

Figure 8 shows the resulting distributions of the magnetic field strength and inclination. An obvious trend is that the contribution of the more inclined field is larger as we integrate longer. The difference is not great for integration up to $2 \mathrm{~min}$, especially for the field strength, but it becomes significant if we integrate longer. If we integrate over $9 \mathrm{~min}$, the distribution of field inclination becomes very narrow with most pixels harboring the horizontal field, while the retrieved mean field strength at $\log \tau=0$ increases by $34 \%$.

\section{Inversions of observed Hinode SP maps}

Previous tests convinced us that the combination of node positions at $\log \tau=0,-0.8$ and -2.0 gives statistically good results, irrespective of the field configurations, so we used this combination for inverting real solar observations. Furthermore, we found out that choosing a PSF with a slightly different amount of defocus does not make a large difference so we used the PSF where a defocus of seven focus steps is included. In order to compare our results to the results of others, we took a standard scan made in normal mode.

Figure 9 shows an inverted part of the normal mode scan used in various studies starting with (Lites et al. 2008) and (Orozco Suárez et al. 2007a,b). Comparison with these studies confirms that the 2D inversions give inverted maps that are similar to those retrieved with other codes, but show much more detail. The network features are finer and the voids containing almost no field are smaller, i.e., not bigger than $5^{\prime \prime}$.

In Fig. 10, we zoom in on the field of view shown in Fig. 2 of Orozco Suárez et al. (2007b) to demonstrate what our code gives on the smallest scales. Magnetic field strength and inclination at $\log \tau=-0.8$ is similar to the maps in Orozco Suárez et al. (2007b) and Asensio Ramos (2009). Fields are predominantly weak with a few stronger features in the intergranular lanes that are still hG. Our maps at $\log \tau=0$, on the other hand, reveal many more features of which some harbor $\mathrm{kG}$ field. The inclination map, at the same time, reveals densely packed mixed polarities over the whole map. Generally, oversampling to pixels size of $0.08^{\prime \prime}$, in case of our 2D inversions, reveals a much finer structure in all the maps.

Figure 11 shows final distributions of magnetic field strength and inclination of internetwork. We filtered out the network contribution by following the procedure from Lites (2011). All the regions where longitudinal apparent flux density exceeds $100 \mathrm{G}$ together with its $2^{\prime \prime}$ surroundings were excluded from the statistics. The retrieved mean field strength at $\log \tau=0$ is around $130 \mathrm{G}$. At the higher layers, the field strength drops as the field becomes more horizontal.

\section{Conclusions and discussion}

An inversion code that self-consistently accounts for the effects that the instrumental PSF has on data, was applied, for the first time, to quiet Sun observations. Extensive testing on MHD 


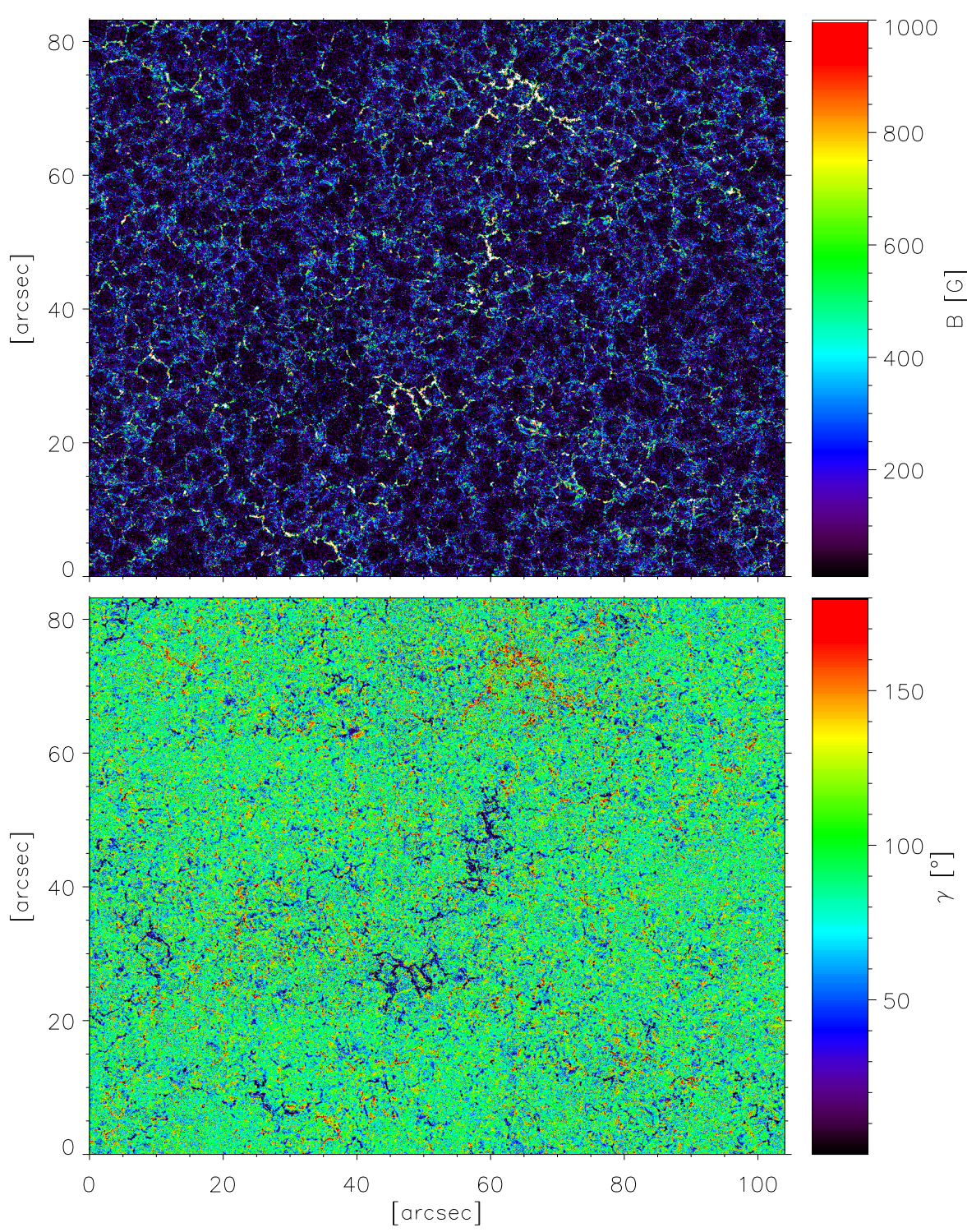

Fig. 9. Results of 2D inversions applied to Hinode SP observations: maps of magnetic field strength (top) and inclination (bottom) at $\log \tau=0$. simulations confirmed that the inverted results make sense and also provided an inversion strategy that we then applied to the solar observations.

In studies like the one presented here, typically only pixels are selected that contain significant signals in $Q$ and $U$ or $Q, U$ and $V$ to ensure that the result returned by the inversion code used to recover the magnetic field strength is reliable. However, this selection disregards signals that in individual pixels are below the noise level, but averaged over a large number of pixels are statistically significant. This not only results in loss of signal, it may also introduce a bias in the results toward the properties that are particular to strong magnetic fields only. The spatially coupled inversion method used here is able to constrain a result using such signals and can, therefore, make use of all pixels in the FOV.

The inversions return a distribution with a mainly weak field, without any secondary peak at kG field strengths, as in Stenflo (2010) and Lites (2011). This distribution shows no peak either at hG values as detected by Orozco Suárez et al. (2007a), but monotonously increases toward the smallest field strengths. This however does not exclude the possibility that real distribution of the field strength does not have a peak at $5-10 \mathrm{G}$ as local dynamo simulations show. The tests on the simulations demonstrate that the code tends to set the field strength to zero when signals are too weak. The code also tends to retrieve mostly horizontal field in the regions that harbor a very weak field. This then produces large differences between the original and retrieved distribution of the field inclinations in the case of local dynamo simulations that show salt and pepper pattern even in these regions.

Owing to the noise, the code tends to overestimate the hG field, which results in a slight overestimation of the mean field strength. Nevertheless the retrieved value comes close to the original. The mean field strength $>100 \mathrm{G}$ at optical depth unity retrieved from the observations puts ours results closer to the results based on the Hanle effect (Trujillo Bueno et al. 2004), although we cannot confirm that the field strength in the upper photosphere is also over $100 \mathrm{G}$, as found by Shchukina \& Trujillo Bueno (2011).

A mean field strength at the solar surface of the same magnitude was also retrieved by Orozco Suárez \& Bellot Rubio (2012) and Bellot Rubio \& Orozco Suárez (2012). Their results, however, show much more horizontal field. Our tests show that this can be explained as an artefact, which is produced by prolonged temporal averaging, since increasing the integration time beyond 

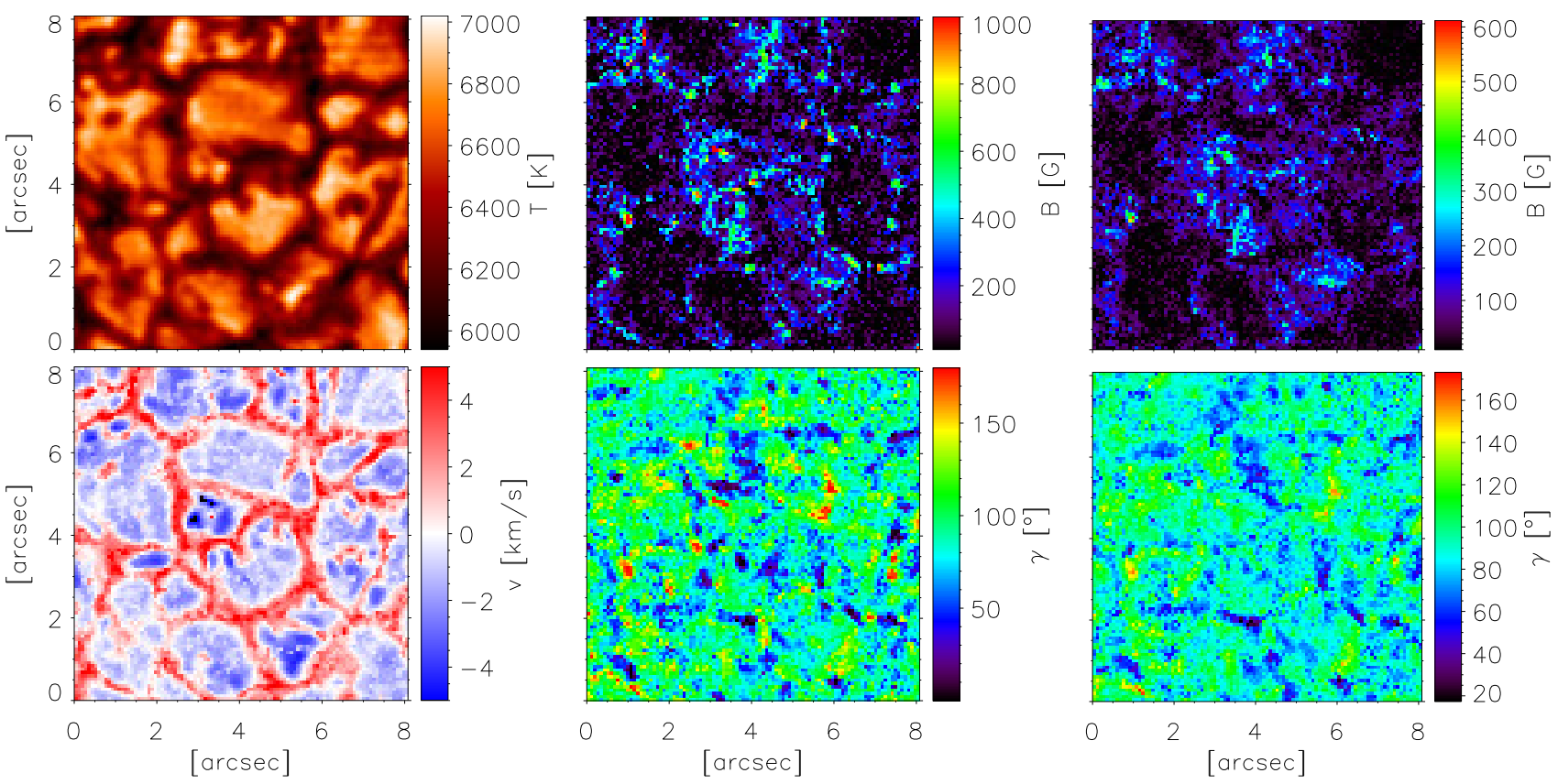

Fig. 10. Results of 2D inversions applied to Hinode SP observations: zoom in to a small field of view (see the text). Top row, from left to right: temperature at $\log \tau=0$ and magnetic field strength at $\log \tau=0$ and -0.8 . Bottom row, from left to right: $\operatorname{LOS}$ velocity at $\log \tau=0$ and magnetic field inclination at $\log \tau=0$ and -0.8 .
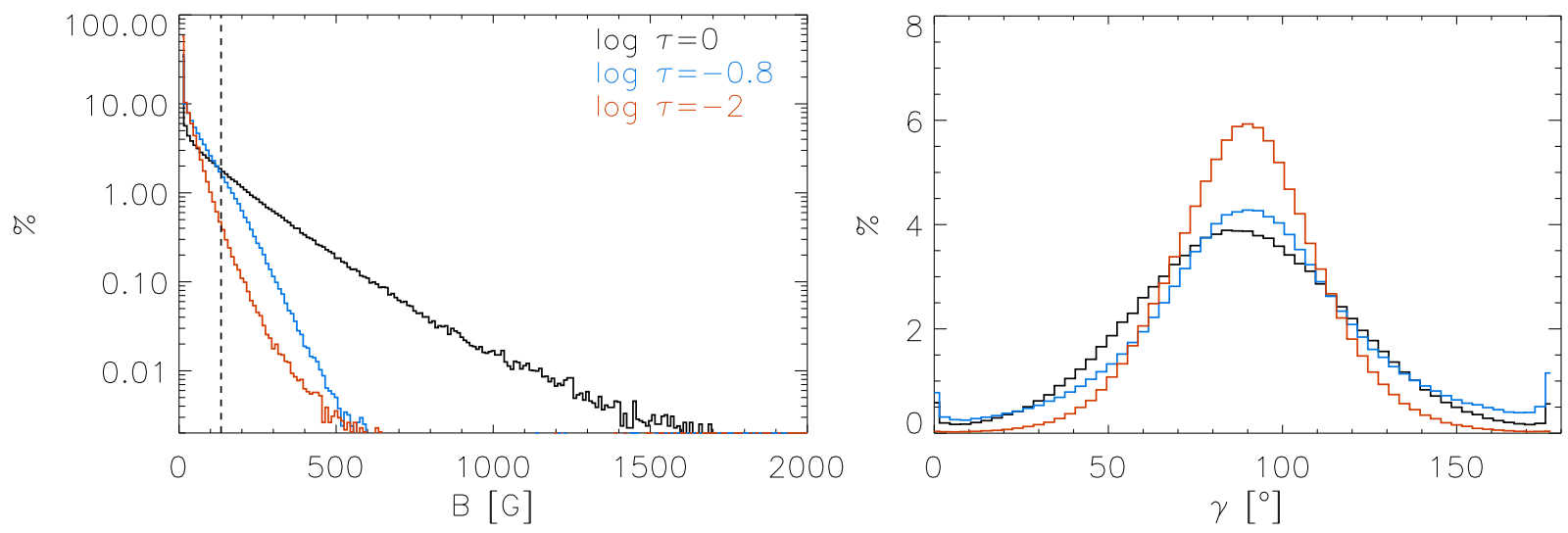

Fig. 11. Results of 2D inversions applied to Hinode SP observations: histograms of magnetic field strength and field inclinations at 3 nodes. Black, blue, and red denote heights of $\log \tau=0,-0.8$ and -2.0 , respectively. Bin sizes are $10 \mathrm{G}$ and $3^{\circ}$. Vertical line indicates the position of the mean field strength at $\log \tau=0$.

the evolution timescale of the solar scene (2-3 min.) artificially increases the apparent contribution of the horizontal fields significantly. In the case in which the distribution of magnetic field is isotropic, this can be easily understood since in that case integration beyond the evolution timescale superposes a statistically independent realization of the magnetic field distribution. This decreases the measured polarimetric signal at the same rate as the photon noise, so that no net improvement of the signal-tonoise ratio can be obtained by continued integration. The scaling properties of the noise equivalent horizontal and magnetic fields, however, continue to favor a more and more horizontally inclined field configuration. At the same time, the granular motions produce wider line profiles, which result in larger retrieved field strengths.

The distribution of the field inclination has a maximum at $90^{\circ}$, which confirms the results given by Orozco Suárez et al. (2007b) and Lites et al. (2008). We cannot, however, interpret this as evidence for a predominantly horizontal field nor can we state that this is in agreement with the results obtained by Asensio Ramos \& Martínez González (2014), who claim that the distribution is quasi-isotropic. As shown in Fig. 5, similar results can be obtained from both predominantly horizontal and a quasi-isotropic distribution of the magnetic field. By reexamining the lower left panel of Fig. 5, one can conclude that even if the photospheric magnetic field is isotropic, it might not be possible to recover this distribution as such. We are currently limited, not only by our inversion tools, but also by the resolution limit of our instruments. Tests of the $2 \mathrm{D}$ inversion technique on the simulated Hinode SP data at the disk center suggest that the information for discerning between the two distributions is just not there.

Acknowledgements. We thank M. Schüssler for snapshots of local dynamo simulations (Sim 1). The National Center for Atmospheric Research (NCAR) is sponsored by the National Science Foundation. We would like to acknowledge high-performance computing support from Yellowstone (http://n2t.net/ ark: /85065/d7wd3xhc) provided by NCAR's Computational and Information 
Systems Laboratory, sponsored by the National Science Foundation. Hinode is a Japanese mission developed and launched by ISAS/JAXA, with NAOJ as domestic partner and NASA and STFC (UK) as international partners. It is operated by these agencies in cooperation with ESA and NSC (Norway).

\section{References}

Asensio Ramos, A. 2009, ApJ, 701, 1032

Asensio Ramos, A., \& Martínez González, M. J. 2014, A\&A, 572, A98

Beck, C. \& Rezaei R. 2015, A\&A, 502, 969

Bellot Rubio, L. R., \& Orozco Suárez, D. 2012, ApJ, 757, 19

Borrero, J. M., \& Kobel, P. 2011, A\&A, 527, A29

Borrero, J. M., \& Kobel, P. 2012, A\&A, 547, A89

Bühler, D., Lagg, A., \& Solanki, S. K. 2013, A\&A, 555, A33

Bühler, D., Lagg, A., Solanki, S. K., \& van Noort, M. 2015, A\&A, 576, A27

Cabrera Solana, D., Bellot Rubio, L. R., \& del Toro Iniesta, J. C. 2005, A\&A, 439, 687

Danilovic, S., Gandorfer, A., Lagg, A., et al. 2008, A\&A, 484, L17

Danilovic, S., Schüssler, M., \& Solanki, S. K. 2010, A\&A, 513, A1

Danilovic, S., Cameron, R. H., \& Solanki, S. K. 2015, A\&A, 574, A28

Domínguez Cerdeña, I., Sánchez Almeida, J., \& Kneer, F. 2006, ApJ, 646, 1421

Frutiger, C. 2000, Ph.D. Thesis, ETH Zürich, Switzerland, Diss ETH No. 13896

Frutiger, C., Solanki, S. K., Fligge, M., \& Bruls, J. H. M. J. 2000, A\&A, 358 1109

Ishikawa, R., \& Tsuneta, S. 2011, ApJ, 735, 74

Keller, C. U., Deubner, F.-L., Egger, U., Fleck, B., \& Povel, H. P. 1994, A\&A, 286,626

Lagg, A., Solanki, S. K., van Noort, M., \& Danilovic, S. 2014, A\&A, 568, A60

Lin, H. 1995, ApJ, 446, 421
Livi, S. H. B., Wang, J., \& Martin, S. F. 1985, Aust. J. Phys., 38, 855

Lites, B. W. 2011, ApJ, 737, 52

Lites, B. W., \& Ichimoto, K. 2013, Sol. Phys., 283, 601

Lites, B. W., Kubo, M., Socas-Navarro, H., et al. 2008, ApJ, 672, 1237

Livingston, W. C., \& Harvey, J. 1975, BAAS, 7, 346

Martin, S. F. 1988, Sol. Phys., 117, 243

Martínez González, M. J., Collados, M., \& Ruiz Cobo, B. 2006, A\&A, 456, 1159

Orozco Suárez, D., \& Bellot Rubio, L. R. 2012, ApJ, 751, 2

Orozco Suárez, D., Bellot Rubio, L. R., Del Toro Iniesta, J. C., et al. 2007a, PASJ, 59, 837

Orozco Suárez, D., Bellot Rubio, L. R., del Toro Iniesta, J. C., et al. 2007b, ApJ, 670, L61

Pietarila Graham, J., Cameron, R., \& Schüssler, M. 2010, ApJ, 714, 1606

Sánchez Almeida, J., \& Lites, B. W. 2000, ApJ, 532, 1215

Sánchez Almeida, J., \& Martínez González, M. 2011, in Solar Polarization 6, ASP Conf. Proc., 437, 451

Schüssler, M., \& Vögler, A. 2008, A\&A, 481, L5

Shchukina, N., \& Trujillo Bueno, J. 2011, ApJ, 731, L21

Socas-Navarro, H., \& Lites, B. W. 2004, ApJ, 616, 587

Stenflo, J. O. 2010, A\&A, 517, A37

Rempel, M. 2014, ApJ, 789, 132

Riethmüller, T. L., Solanki, S. K., van Noort, M., \& Tiwari, S. K. 2013, A\&A, 554, A53

Tiwari, S. K., van Noort, M., Lagg, A., \& Solanki, S. K. 2013, A\&A, 557, A25 Trujillo Bueno, J., Shchukina, N., \& Asensio Ramos, A. 2004, Nature, 430, 326 van Noort, M. 2012, A\&A, 548, A5

van Noort, M., Lagg, A., Tiwari, S. K., \& Solanki, S. K. 2013, A\&A, 557, A24

Vögler, A. \& Schüssler, M. 2007, A\&A, 465, L43

Vögler, A., Shelyag, S., Schüssler, M., et al. 2005, A\&A, 429, 335 